\title{
Des traces du II moi " professionnel dans le discours d'enseignants de FLE : apports des entretiens d'autoconfrontation
}

\author{
Traces of the professional 'me' in FLE teacher discourse: contributions of \\ self-confrontation interviews
}

\section{Estefanía Domínguez}

DILTEC-IDAP, Université Paris 3-Sorbonne, France

\begin{abstract}
Résumé : II s'agit dans cet article de mettre en exergue ce que des discours d'enseignants sur leur propre activité enseignante apportent en termes de découverte du « moi » professionnel. Pour ce faire, nous nous basons sur des discours d'enseignantes de français langue étrangère ${ }^{1}$. Au regard de leurs propos, nous montrerons comment les dynamiques discursives permettent de déceler des paramètres constitutifs de leur pratique enseignante. Nous verrons que les informatrices se (re)découvrent au travers de leurs image, dire et faire, montrant alors des conceptions assumées de leur enseignement mais aussi une partie de leur pratique qui leur échappe dans le vif de l'action, notamment leur voix et leur regard.

Mots-clés : agir professoral, discours enseignant, autoconfrontation, réflexion, style.
\end{abstract}

\begin{abstract}
This article investigates how teachers' pedagogical speeches are able to contribute to the notion of exploring the professional "self". The article concentrates on the speeches of teachers of French as a Foreign Language ${ }^{2}$. It will show how the discursive dynamics of their words reveal the constituent elements of their teaching practice. We will see that the informers (re)discover themselves through their image, words and actions, not only allowing them to show definite conceptions of their teaching but also to explore parts of their practice that elude them in the heat of the moment (such as their voice or look).
\end{abstract}

Keywords: teaching behaviour, teaching speech, self-confrontation, reflection, style.

\section{Introduction}

Notre étude s'inscrit dans le domaine de la didactique des langues et plus précisément dans celui de la didactique du FLE. Nous nous intéressons à l'une des pratiques de transmission dont use l'enseignant à savoir, l'activité de prescription entendue comme "l'activité langagière [...] qui incite à l'action et vise la transformation de l'autre et, en l'occurrence, de ses savoirs et savoir-faire langagier dans la classe dans la langue étrangère [et qui] se déploie en injonctions (dire de faire), en consignes liées à une tâche (dire quoi faire) ou en instructions (dire

\footnotetext{
1 Par la suite, français langue étrangère sera désigné par FLE.

2 From then on, French as a Foreign Language will be mentioned as FLE.
} 
comment faire et pourquoi faire) " (Rivière, $2008: 51$ ). Cette activité est de nature multimodale $^{3}$ car elle imbrique la parole, le regard, les gestes, etc. et est plurisémiotique car sa gestion peut également appeler des ressources matérielles (tableau, documents, etc.). L'activité de prescription est également interactionnelle car elle s'actualise dans le contexte de la classe entre l'enseignant et les apprenants.

La notion-cadre de ce travail est l'agir professoral défini comme «l'ensemble des actions verbales et non-verbales, préconçues ou non, que le professeur met en place pour communiquer des savoirs à un public donné dans un certain contexte $"$ (Cicurel, 2011 : 7). L'activité de prescription est un révélateur de cet agir (Domínguez, 2013) et permet entre autres de structurer les enseignements et les apprentissages. Afin de mieux comprendre cette activité, nous avons donné la parole à trois enseignantes pour les amener à mettre des mots sur cette partie de l'agir professoral et voir dans quelle mesure elles sont conscientes de la multimodalité prescriptive.

Dans cet article, nous nous focaliserons sur les verbalisations qui illustrent des éléments singuliers ou ce que nous appelons des "traces du moi ». Nous montrerons alors que lors de la mise en mots sur l'activité réalisée et filmée, apparaissent toute une série de catégorisations. Nous nous proposons donc de dégager du flux verbal, souvent confus et haché, les moments où les enseignantes se découvrent ellesmêmes - avec étonnement quelques fois - se définissent, se critiquent, etc.

\section{L'autoconfrontation comme clé d'accès à l'agir professoral}

\subsection{Les verbalisations sur l'action : de l'observable et de l'inobservable}

Comme le signale Habermas (cité par Cicurel, 2011 : 116), "l'action ne se désigne pas d'elle-même, mais son sens peut être éclairé par celui qui l'a accomplie ॥. Dans le cadre des études sur le métier qui nous occupe, l'accès aux paroles d'enseignants permet « d'éclairer leur agir enseignant et les modalités de constitution du répertoire didactique ${ }^{4}$ qui sous-tend cet agir" (Bigot et Cadet, 2011: 22) et favorise en conséquence la mise en lumière de la "part inobservable " (Ibid.). En d'autres termes, il s'agit de ne pas se limiter à l'interprétation du chercheur qui analyserait les séances de cours filmés mais "d'aller chercher le point de vue des acteurs" (Ibid. : 12). Les commentaires sur l'action revue permettent également de faire émerger la face cachée de l'action, ce que ressentent les acteurs, ce qu'ils pensent, les décisions qu'ils ont prises, etc. Pour avoir accès à cette part inobservable, la vidéoscopie fait partie des pratiques scientifiques qui permettent aux enseignantsparticipants de mettre des mots sur leur action (action effective, effectuée et revécue). Dans le cadre des recherches sur l'agir professoral, l'acteur est invité à commenter des cours ou passages de cours (filmés préalablement) lors d'un entretien dit d'autoconfrontation (Clot, 2001).

\footnotetext{
3 Nous entendons cette multimodalité comme une multicanalité où les aspects verbaux et non-verbaux sont à entendre comme synergiques (Cosnier, 1997 : 2).

${ }^{4}$ Le répertoire didactique est défini «comme un ensemble hétéroclite de modèles, de savoirs, des situations sur lesquels un enseignant s'appuie. Ce répertoire se constitue au fil des rencontres avec divers modèles didactiques [...], par la formation académique et pédagogique auquel il a été exposé, par l'expérience d'enseignement qui elle-même modifie le répertoire." (Cicurel, 2002: 157). Voir également Cadet, 2004.
} 


\subsection{Quelques précautions}

Quelques précautions doivent être signalées car les discours sont produits dans un contexte précis et émane du sujet à un instant " $\dagger$ ", il y a donc également une forte part de subjectivité. Malgré cela, nous ne pouvons nier l'accès à une certaine part de vérité car «la subjectivité et l'intersubjectivité sont considérées comme des moyens incontournables de construction de savoirs et non comme des obstacles à la production de connaissances " (Anadon et Guillemette, 2007 : 3). Par ailleurs, comme le signale Cicurel (2015:43):

Le but de l'expérience est d'aller au plus près de ce qu'on pourrait appeler la pensée-enseignante-en-action. Eł en effet, le flux de paroles que provoque le visionnement de l'action d'enseigner est révélateur d'une partie de ce qui habite l'univers intérieur du professeur [...] Les verbalisations ne sont pas la «réplique» des situations originales de classe de langue mais "la relation qui en est faite».

C'est la manière dont les acteurs se représentent l'action accomplie qui est porteuse de sens.

Il y a aussi le statut de l'enseignant qui se trouve affecté par la situation d'autoconfrontation. En effet, il change de cadre et passe en quelque sorte "d'acteur sur le terrain, tenant un rôle prédéterminé auprès de ses élèves [à] observateur de lui-même [car] sollicité pour produire de la signification à propos de ses actions [et devient] chercheur dans le contexte " (Cicurel, 2011 : 250-251). Le chercheur doit donc composer avec ces paramètres.

\section{Présentation du corpus}

Notre réflexion s'appuie sur un corpus de discours à caractère réflexif constitué en 2012. Nous avons filmé et retranscrit quatre heures de cours de FLE chez trois enseignantes expérimentées ${ }^{5}$ qui exercent dans un centre universitaire en France. Le public des cours était composé d'apprenants internationaux de niveau B2.

Les entretiens d'autoconfrontation se sont déroulés sur le lieu de travail des enseignantes. Comme notre recherche portait sur la passation de consignes, nous avons préalablement défini les passages qui s'y référaient. Ces extraits étaient ensuite soumis aux enseignantes en entretien individuel. En nous inspirant de l'entretien d'explicitation (Vermersch, 1994), nous leur avons donc demandé de mettre des mots sur leur action sans poser de questions en "pourquoi $\|^{6}$. Le thème de la recherche n'a volontairement pas été dévoilé aux informatrices afin de ne pas influencer leurs discours rétrospectifs. Cependant, nous avons intégré en fin d'entretien un outil de l'entretien non-directif à savoir, une question ouverte qui explicite l'objet de la recherche?.

\footnotetext{
5 Pour des raisons pragmatiques, nous traiterons essentiellement des extraits de discours de Cécile.

6 D'après Vermersch, "ce style de question oriente l'attention sur les raisons d'un acte et non sur sa description, et induisent un travail de raisonnement, de réflexion » (2004 : 4). Nous voulions donc éviter d'induire le conscientisé.

7 La question posée de type non-directif est la suivante : « est-ce que tu as reconnu des actions qui font
} 


\section{Les traces du moi : entre affirmation du ॥ je " professionnel et découverte de soi}

Yves Clot précise que "passer l'action au crible de la pensée est une action à part entière; une action sur soi qui ne laisse pas la première action, devenue objet de pensée en l'état» (2001 : 256). Dans les discours produits par des enseignants face à leur action filmée, il existe de nombreuses alternances entre des modalités descriptives, argumentatives et évaluatrices sur ce qui est dit ou fait, et qui, comme nous le verrons, créent et montrent des prises de conscience, des (re)découvertes de soi au moyen de la production, construction et reconstruction des discours. L'activité en elle-même ne laisse pas non plus l'enseignant "indifférent ", il entre en discussion avec son propre «moi ». À cet égard, les hésitations et les émotions imprègnent leur parole. Brahim Azaoui signale que "l'autoscopie est une première étape dans la découverte de soi. En dépit du choc (Linard, Prax, 1978) que la confrontation à son image peut entraîner, cette démarche facilite la "posture réflexive" (Schön, 1994) nécessaire à une prise de conscience de sa pratique " (2014: 2). Mais comment se manifestent ces prises de conscience ? Comment celles-ci sont-elles vécues? Quelles traces observables pouvons-nous repérer dans les discours rétrospectifs?

\subsection{Les discours réflexifs : entre (re)découverte et évaluation}

Comme nous l'avons déjà signalé, se voir faire cours n'est pas une tâche habituelle chez les enseignants. Ainsi, les discours produits prennent souvent la forme de jugements où l'acteur est partagé entre ce qu'il découvre et ce qu'on lui demande : se voir et parler.

L'informatrice Cécile, lors de la question ouverte finale, établit un bilan ponctué de constats et d'appréciations :

la mise en place de consigne [...] c'est moi aussi en gestuelle j'ai toujours je sais pas pourquoi enfin c'est un truc faudrait que je travaille là-dessus :: j'ai toujours la tête un peu baissée je sais pas pourquoi comme si j'avais honte de présenter une consigne ça paraît ridicule c'est mais ouais je sais pas ça fait partie de moi je : je dois être comme ça euh ::: j'ai toujours euh ::: je pense que je fais ::: bon voilà.

Dans ce passage, nous pouvons apprécier une catégorisation « c'est moi aussi en gestuelle ", ce qui la définirait donc en matière prescriptive. Par ailleurs, son discours montre une prise de conscience facilitée en partie par la mise en mots; discours hésitant parsemé de «je sais pas », "enfin », «je sais pas pourquoi ». L'enseignante se découvre sous un angle extérieur et prend conscience de comment son corps se meut au moment de la passation de consignes "j'ai toujours la tête un peu baissée ». Nous pouvons remarquer un certain tiraillement entre la découverte de soi et la recherche d'une explication et même d'un besoin de solution. Elle émet des

partie de ta pratique quotidienne en ce qui concerne la consigne ? ॥. Elle porte donc sur la manière dont les enseignantes agissent en matière de passation de consignes. Signalons que l'objectif général était de compléter les données et d'enrichir la compréhension de l'agir prescriptif chez chacune des enseignantes; l'objectif précis étant de voir la place du verbal et du non-verbal en matière de passation de consignes. 
appréciations ("je ne sais pas pourquoi», "ça paraît ridicule ») et assume partiellement sa caractéristique «je sais pas ça fait partie de moi ». De son constat émerge un début de solution ("il faudrait que je travaille là-dessus ») mais elle n'arrive pas à conclure et, suite à quelques hésitations, elle marque une rupture "bon voilà " donnant l'impression ne de pas être satisfaite ou de ne pas avoir fait le tour de la question. Elle poursuivra son discours sur sa technique de passation de consignes qui elle, est maitrisée, consciente et réfléchie et fait partie d'un savoir pratique acquis par expérience (cf. plus bas, les extraits du point 3.1.3).

Les jugements émis par les enseignantes sont souvent évaluatifs. A ce sujet, Isabelle en tout début d'entretien, lors des premières minutes d'exposition à son image, déclare "je remarque que je parle au tableau (rires) que j'ai donné la consigne en écrivant et ça je sais que c'est pas euh que les étudiants peuvent avoir des problèmes pour m'entendre justement donner la consigne ». Elle prend donc conscience de ce qu'elle fait, vécu verbalisé par "je remarque que " et, comme pour le cas de Cécile, une marque d'hésitation ponctue son discours «je sais pas que c'est euh que ॥. Un peu après dans le discours, l'enseignante s'attardera sur sa voix "j'ai l'impression de beaucoup hacher les mots en fait [...] je sépare les mots ॥, la vidéo agit comme un véritable miroir qui lui permet de percevoir des éléments prosodiques de son agir professoral.

En ce qui concerne Victoria, elle s'engage dans un discours qui passe de la découverte ou redécouverte de la « matérialité » de sa voix :

hum ++ les consignes euh pff ++ ma voix/ce que je remarque et bon je le
savais déjà plus ou moins mais c'est que je:: j'ai pas une voix qui por:::.te je:::
j'essaye d'accentuer certains mots:: euh: en fonction de ma fatigue aussi +
personnelle tout simplement en fonction des moments du cour j'ai une voix
plus monotone euh je SAIS que c'est un défaut d'ailleurs dans ce cour là dès
le premier cours il y a un étudiant qui m'a dit euh pouvez-vous parler plus
fort + c'était la rentrée ça faisait six mois que je n'avais pas enseigné (rires)
fallait que je réadapte ma voix je mettais dit oula bon en effet j'ai intérêt
donc à faire attention (respire) mais je sais $\uparrow$ que j'ai la voix qui descend très
rapidement sur la fin des des consignes et là je je je m'en suis rendu compte
ca Pose problème parce que ++ parce que XX l'étudiant peut se dire ben
c'est pas important + si elle le dit si bas c'est que ben c'est ::: c'est même
pas audible parfois peut-être $\uparrow$ j'en sais rien ben c'est que c'est pas
intéressant (bruit de bouche) j'essaye d'y faire attention mais c'est pas
toujours évi- facile parce que c'est un effort vraiment + physique du coup
pour moi + euh:::: euh:::: sinon: pour la gestion du tableau

Comme le signalent Clot et Faïta, "la prise de conscience n'est donc pas la découverte d'un objet mental inaccessible auparavant mais la redécouverte - la recréation - de cet objet psychique dans un nouveau contexte qui le "fait voir autrement"' " (2000: 34). Dans l'extrait, nous observons que la décentration de soi permise par la vidéoscopie remet au premier plan un élément problématique de la pratique quotidienne de l'enseignante, la voix. Sa propre voix qu'elle entend lui fait dans une certaine mesure défaut, d'autant plus pour l'activité de passation de consignes où l'attention que portent les apprenants est conditionnée, entre autres, par une voix qui devrait être claire et audible. Nous avons accès ici à une partie de I'histoire interactionnelle de Victoria et son groupe d'apprenants (un étudiant lui avait fait part d'une remarque sur sa voix) mais aussi au fait qu'elle s'était déjà 
interrogée sur sa voix. Nous voyons donc tout un pan qui serait de l'ordre de l'inobservable pour le chercheur s'il n'y avait pas eu d'activité de verbalisation. Pour l'enseignante, il s'agit d'une redécouverte d'une partie du soi accompagnée par une prise de conscience, repérable par des marqueurs linguistiques tels que «je remarque " ou "je m'en suis rendu compte ». Le rôle de l'autoconfrontation dans cette conscientisation est clairement manifestée par la déclaration suivante « là je je je m'en suis rendu compte ça POSE problème ॥.

Les extraits de discours produits par les enseignantes que nous venons de présenter sont pour la plupart appréciatifs; nous pourrons noter aussi une certaine surprise de leur part. À titre d'exemple, Isabelle déclare "je remarque que je parle au tableau " dans une phrase exclamative dont la prosodie marque l'étonnement. Dans le vif de l'action, prise par plusieurs foyers d'attention, son orientation et déplacement du corps passent probablement au second plan. Également dans une découverte de l'action qu'elle mène, cette informatrice prend conscience d'une certaine incohérence entre ce qu'elle dit qu'elle va faire et ce qu'elle fait réellement. II s'agit d'un moment du cours où elle énonce les consignes avant de visionner un extrait de film, elle donne donc une première consigne en demandant aux apprenant de se focaliser sur quelques éléments généraux (le métier du protagoniste, le lieu de l'action, etc.) en énonçant "je ne vous dis rien » mais elle ajoute d'autres informations qu'à priori elle n'était pas censée donner. Lors de l'entretien, elle fait alors part de son ressenti : "c'est drôle pour moi parce que je dis je vous dis RIEN puis en fait je dis plein de choses" tout en accompagnant ces paroles d'une mimique faciale entre le rire et la surprise, suivis d'un silence, comme si sa réflexion continuait dans son for intérieur. Le mouvement auquel amène l'autoconfrontation fait que "le sujet découvre [des éléments de] son activité »; cela le mène entres autres, "à se mettre par la force des choses à distance de luimême, de se considérer comme l'acteur en partie étranger de sa propre action $"$ (Clot et Faïta, 2000 : 26).

\subsubsection{Les discours réflexifs: le moi émergeant ou reconnu}

Les verbalisations issues de l'autoconfrontation permettent également l'accès à des éléments qui montrent chez les enseignants un certain style ou profil qui trouve sa place à l'intérieur du genre professionnel, dans notre cas dans le métier enseignant. Pour Clot et Faïta, le genre est « ce que les travailleurs d'un milieu donné connaissent et voient, attendent et reconnaissent et redoutent; ce qui leur est commun [...] c'est comme "un mot de passe " connu seulement de ceux qui appartiennent au même horizon social et professionnel ॥ (2000: 11). Les enseignants se reconnaissent dans leurs objectifs, l'agir professoral regorge d'action verbales et non verbales connues et reconnues: les échanges ternaires ${ }^{8}$, la gestion de la parole grâce au regard et aux gestes, la passation de consignes, commencer et finir un cours, etc. À l'intérieur de ce genre reconnu, l'enseignant dispose d'une marge de manœuvre, d'une façon de faire qui lui est propre. Ainsi, "chaque sujet interpose entre lui et le genre collectif qu'il mobilise ses propres retouches du genre. Le style peut donc être défini comme une métamorphose du genre en cours d'action ॥ (Clot et Faïta, 2000 : 14). Cette façon de faire qui lui est propre met au premier plan la singularité de la

8 Organisées comme suit : question, réponse et validation. 
personne même si certains peuvent revendiquer une coupure entre le "moi» personnel et leur " moi» professionnel; cependant la personnalité peut toujours déteindre dans le vif de l'action. Cicurel pointe cette singularité tout en émettant des réserves sur le terme de "style ». Elle invite à considérer les enseignants " comme auteurs de pratiques [celles-ci étant] identifiables et observables et, qu'à ce titre, il est envisageable de dire qu'un professeur agit dans un certain style $"(2011$ : 148). Nous allons donc à présent illustrer des marques de "style " ou en d'autres termes des façons de faire propres aux enseignants qui pourront être énoncées ou encore assumées et revendiquées.

L'enseignante Cécile se définit souvent et revendique d'ailleurs son côté spontané et l'usage du rire comme partie intégrante de son métier mais aussi d'ellemême. Lors d'une activité de débat, elle explique des règles bien précises concernant l'utilisation de chapeaux de différentes couleurs; chaque couleur étant associée à un type d'argumentation. Le discours qui porte sur ce passage est le suivant:

\begin{abstract}
n'importe quoi ça me fait rire de me voir +++ donc euh dans un premier temps je je me marre parce que je:: je suis toujours dynamique par rapport à mes/enfin $\mathrm{MOI}$ ça/je suis toujours hyper enthousiaste de mes activités donc euh c'est bien c'est bon voilà ça me fait rire ALORS dans un premier temps je suis en train de euh:: leur expliquer COMMENT on va organiser le débat en termes:: LOGISTIQUE [...] ensuite il y a euh:: une explication PRATIQUE [...] par rapport à euh la couleur " [...] puis je leur ai donné des exemples pour bien voir la différence parce que $\uparrow$ c'est/je je parce que je l'ai vécu ce n'est pas toujours très clair donc je je je M'EFFORCE de:: donner des exemples pour qu'ils comprennent la différence entre une idée réaliste une idée fantaisiste euh voilà je donne quelque chose qui est possible et l'autre qui est complètement FOU euh:: évidemment je passe par l'humour parce que c'est MOl:: donc euh je/ et puis ça les fait rire je leur dit que celui qui a le chapeau jaune il est fou il est mala::de enfin bon bref $\downarrow$ donc euh je $X X$ je passe par l'humour en général quand je quand je donne des exemples.
\end{abstract}

L'impact émotionnel de l'action en image peut être défini chez Cécile comme positif, sa mimo-gestuelle faciale est détendue, elle sourit et son discours est plutôt fluide. Ses premiers mots montrent une certaine surprise mais également une certaine satisfaction. En effet, il y a une sorte de bien-être qui est présent dans tout l'extrait, comme si le fait de se "voir faire» la réconfortait et permettait une approche d'un des paramètres qui la définissent : I'humour. L'enseignante se définit comme "dynamique " et "enthousiaste " et insiste, à l'aide d'un adverbe, sur sa caractéristique "évidemment je passe par l'humour ». Ce trait assumé et revendiqué est accentué par une énonciation marquée par une association à sa personne, elle déclare alors " C'est MOl » en insistant sur le pronom tonique.

Notons que ce passage s'inscrit dans une "production fictionnelle " (Cicurel, 2011 : 68) que l'enseignante réalise pour donner un exemple. En effet, elle va en quelque sorte théâtraliser une situation en imitant quelqu'un qui défendrait l'utilisation sans contrôle du papier en ignorant et en ne se préoccupant pas de l'impact de ses actes sur la planète. Pour ce faire, elle n'économise pas ses gestes, ses mimiques, ses déplacements ni le ton utilisé. Son enthousiasme et humour 
revendiqués sont également énoncés lorsqu'elle met des mots sur le passage où elle finit d'expliquer le rôle et la signification de chaque chapeau :

\begin{abstract}
$\mathrm{AH}$ et je leur dis après ben pour moi je suis oui oui + sur la FIN parce que quand je voilà à la fin de ma consigne pour euh lancer:: euh je dis souvent c'est parti on y va allez-y blabla donc j'ai toujours mes petites formules et c'est vrai que::moi je rajoute fantastique parce que voilà ça me plaît je sais que que ça va me plaire de les voir faire ça donc c'est aussi une un moyen de EUX ça les fait sourire euh d'ailleurs on le voit là mais:: euh ils savent euh c'est un moyen de leur montrer que moi je suis HYPER enthousiaste pour faire cette activité s'il y avait que moi je la ferais tout de suite donc euh/ ben ca les met en: conditions euh d'enthousiasme si si on peut dire quoi++ hum.
\end{abstract}

Ici, les "petites formules» ont comme objectif non seulement de dynamiser la classe mais aussi de créer un climat favorable marqué par la bonne humeur. Dans son discours, nous pouvons observer la prise en compte du pôle apprenant qui soustend son action (" c'est aussi une un moyen de EUX ça les fait sourire ", "ça les met en: conditions euh d'enthousiasme »). Cécile ajoute un dernier commentaire qui porte sur le moment où elle lance l'activité :

\begin{abstract}
et puis VOILA et + à la fin l'éternel euh mot de bon courage bon débat amusez-vous bien moi j'y crois (rires) donc euh je les encourage à ma façon mais ça reste très :: bon ça reste bon genre mon dieu on va jamais y arriver AU CONTRAIRE c'est + c'est GENIAL quoi éclatez-vous.
\end{abstract}

Nous observons que Cécile, par son attitude et son comportement, espère que les apprenants puissent s'identifier et agir de la sorte. Ajoutons que le climat instauré par l'enseignant en cours de langue est d'autant plus essentiel lorsqu'il s'agit de mener des activités de production orale car les apprenants sont invités à réagir et à interagir en langue étrangère, ce qui suppose de surcroît une exposition de leur corps, de leur voix et de leur discours en français. Cécile manifeste clairement cette volonté de dynamisation et de motivation "je les encourage à ma façon ", "c'est génial quoi éclatez-vous ». Nous pouvons signaler que quand l'enseignante se voit faire et verbalise cette action, elle revit, dans une certaine mesure, le sentiment de joie qu'elle éprouvait à l'instant même du cours.

\title{
3. 1.2. Construction du «moi » prescriptif dans la reconstruction de l'activité
}

La reconstruction de l'expérience vécue, facilitée par le visionnage, permet également d'observer certaines conceptualisations en acte. Dans le cadre de la présente recherche, cette conceptualisation peut être enclenchée et favorisée par la mise en mots. Cependant, lors de la question ouverte finale, qui agit en quelque sorte comme bilan, l'émergence d'un profil prescriptif est sensé apparaître ou du moins la mise en exergue de paramètres qui entreraient en jeu lors de la passation de consigne. Cette étape finale, rappelons-le, se déroule au moment où l'enseignante vient d'être plongée dans son image, son agir, ses réflexions, ses critiques, ses prises de conscience, ses affects, etc. Quels sont les résultats de cette immersion visuelle, psychique et émotionnelle-dans le "moi enseignant»- sur le «moi prescripteur»? 
Cécile s'engage dans une explicitation de sa pratique prescriptive à travers laquelle nous avons accès aux paramètres constitutifs de sa pratique. Lors de cette mise en mots, elle illustre certaines façons de faire avec des exemples issus des extraits visionnés mais aussi avec d'autres moments de sa pratique professionnelle, ce qui montre une articulation, des va-et-vient entre le revécu et sa pratique quotidienne :

je donne euh :: d'abord c'est plus une organisation dans ma consigne voilà comment qu'est-ce que j'ai prévu moi en terme de parcours pédagogique j'ai l'impression voilà je vous explique ce qu'on va faire c'est une explication de ce qu'on va faire d'abord ça passe par là donc ça c'est ma façon de faire je vous explique ce qu'on va faire ++ pour que vous puissiez vous situer dans le contexte de + aujourd'hui la classe ++ et à partir de là + je vous dis :: bah + puisque je vous ai expliqué ça on va travailler avec ça donc là vous allez à tel endroit dans le livre par exemple si c'est dans un livre + où vous allez euh me donner euh où vous allez faire euh la description mais je je je PRECISE un peu plus en rentrant dans le détail pratique.

Nous observons un début de discours marqué par la locution adverbiale (d'abord) qui permet de montrer que l'enseignante va s'engager dans une description qui comporte une gradation ou plusieurs étapes. Nous avons donc accès à un premier palier de " contextualisation situationnelle " (Rivière, 2006: 439) sous-tendu par les objectifs didactiques et pédagogiques visés, qui seront atteints grâce à l'activité proposée. Cette "explication de ce qu'on va faire " joue un rôle capital pour Cécile et fait d'ailleurs partie de ses procédés, ce qu'elle manifeste avec "c'est ma façon de faire ». Suite à cette étape, vient la "spécification des tâches" (Ibid.: 441) marquée dans sa verbalisation par "à partir de là ". Nous remarquons comment son discours d'abord distancié de l'action se plonge ensuite dans un discours qui se rapproche de l'interaction de la classe, ce que nous observons au travers de l'utilisation du pronom "vous", "je vous explique ", "pour que vous puissiez $॥$, etc. Nous avons donc ici accès à un discours qui donne à voir sa conception de la passation de consignes, qui semblerait être un rituel acquis par expérience.

Ensuite, l'enseignante décrit la transmission concrète de consignes, le moment où elle les énonce aux apprenants :

: je::.:: je formule la consigne et je vais toujours la répéter ++ d'une autre façon je vais la reformuler en fait + parce qu'elle est/parce qu'elle si $\mathrm{MOI}$ elle me paraît claire + elle ne l'est pas forcément euh de enfin pour les étudiants pour eux c'est pas forcément clair donc je la reformule ++ parfois je suis obligée parce que peut-être que c'était pas assez clair ou peut-être parce que l'étudiant est parti dans son imagination de + recentrer donc tout à l'heure on a bien vu avec l'étudiante qui disait est-ce que je dois lire l'article + non j'ai dit on décrit donc je recentre ++ euh:: hum::

Nous pouvons constater que chez Cécile, la formulation de consignes est étroitement liée à la reformulation de celles-ci. Nous avons donc accès à une certaine conviction pédagogique qui d'ailleurs est vérifiée dans la première partie de l'entretien d'autoconfrontation lorsque Cécile déclare "donc ÇA c'est + la répétition de consignes et qui est:: si + chez moi elle est systématique parce que:: (en riant) je connais bien mes étudiants et TOUT le public je veux dire euh + je le ferais $\uparrow$ 
avec n'importe qui ». L'activité de reformulation est donc manifestée, assumée voire même revendiquée.

Ensuite, l'enseignante énonce une autre composante (introduite par "après ») de son activité prescriptive à savoir, la vérification:

et puis après je vais m'adresser $X$ donc ça ça va être c'est ma façon de faire par rapport à au groupe classe et après c'est vrai que je je vais euh:: comme je me déplace + j'aime bien me rendre compte de:: euh enfin je vais faire une vérification est-ce que ma consigne a été comprise donc vérification qui va se passer soit en déplacement donc je l'ai fait dans la première séquence pour voir s'ils ont bien reçu sur le fichier s'ils savent ou s'ils peuvent l'ouvrir donc vérification que la consigne a été reçue et:: comprise euh:: vérification qu'ils sont en train d'appliquer la consigne.

Si la contextualisation situationnelle, la spécification des tâches et la reformulation sont des étapes observables et verbalisées en situation d'interaction, la phase de vérification :

- apparaît comme validation des étapes précédentes et

- appartient à l'enseignante et se réalise lors d'une prise de distance (les apprenants se mettent au travail).

L'étape de vérification est notamment liée aux ressources non-verbales dont dispose tout enseignant, c'est effectivement par le truchement de ses déplacements que l'enseignant contrôle (exemples: la compréhension, l'attention, etc.) et ce, grâce au regard qu'il porte sur le groupe. À cet égard, l'intervalle temporel est important car c'est précisément «quand un moment de silence advient, que [l'activité attentionnelle de l'enseignant] est allégée, il peut [alors] passer en revue son action, son discours et l'action des apprenants [...] il met en relation par un processus de traitement de l'information, l'état de la mise au travail et son projet ॥ (Domínguez et Rivière, 2014).

Enfin, nous avons affaire dans le discours de Cécile à une synthèse de son activité prescriptive que nous pouvons envisager comme le fruit de l'activité d'autoconfontation, suivie d'une mise en forme (ou mise en articulation) des paramètres constitutifs de sa pratique (extraits précédents) et d'une éventuelle volonté de clarification. Cette dernière étape reprend les différents paliers de son activité prescriptive développés plus haut :

mais OUI ça va être toujours mon fonctionnement en fait euh c'est un peu l'organisation du cours, la consigne, la reformulation, et derrière l'explication si nécessaire ou la vérification la vérification de toute façon au niveau du visuel je vérifie toujours que les étudiants ont bien compris la consigne il y a un des coups de tête hein ça suffit ou des yeux qui partent + des yeux avec des froncements de sourcils pour moi ça me suffit je je du coup je reformule donc je le fais:: j'ai un regard sur les étudiants je PORTE un regard je pense sur les étudiants pour euh parce que j'ai besoin de euh ben j'ai besoin de savoir si j'ai été claire et je ne le suis pas toujours hein c'est pas évident de + toujours bien formuler en plus euh (respire) parfois on a un truc technique qui est très clair et puis parfois c'est pas si clair que ça euh ++ ouais voilà c'est à peu près ça. 
Comme nous pouvons le voir, pour ce qui est des étapes qui composent l'activité de transmission de consignes, son discours est structuré et clair. Nous avons ici un exemple de l'emboîtement discursif propre aux discours produits en autoconfrontation'. À ce sujet, nous pouvons observer comment d'un discours de synthèse (où Cécile résume sa façon de faire prescriptive) et suite auquel nous pourrions attendre une " fin », l'enseignante rebascule dans un discours explicatif qui se focalise sur les raisons pour lesquelles elle reformule les consignes (« il y a des coups de tête ", "des yeux qui partent»). Nous pourrions considérer qu'elle théorise sa pratique, qu'elle attribue un rôle spécifique au regard en essayant de le nommer ; c'est en approfondissant sa description qu'elle précise concrètement "j'ai un regard sur les étudiants " en se reprenant à l'instant même, comme dans une prise de conscience instantanée marquée par l'intensité du verbe lorsqu'elle dit «je PORTE un regard $»$. Comme le signale Cicurel (2015 : 47-48) :

Dans le processus de conceptualisation à partir de l'action, le professeur change en effet de point de vue. Une fois que le praticien n'est plus engagé dans l'action, il adopte une " posture réflexive ». En se regardant faire, il se transforme en un commentateur l'action. N'étant plus absorbé par les tâches d'enseignement, il reconstruit sa pratique et, à ce titre, prend une distance vis-à-vis de l'action. En effet, on le voit vouloir tendre vers une "position d'expertise ", il décrit ce qu'il fait et en donne les raisons, il énonce des normes de comportement.

Au travers des extraits analysés nous avons tenté de montrer comment se manifeste cette "posture réflexive" et en quoi elle permet l'accès à une manifestation du « moi ». La reconstruction de la pratique a permis à Cécile, comme nous l'avons vu, de donner à voir une « matrice » de son activité de consignes.

\section{Conclusion}

Dans la salle de cours, l'enseignant est acteur, en situation d'autoscopie, il devient spectateur et commentateur. Les représentations du «moi » enseignant existent en dehors de l'autoconfrontation; cependant cet outil encourage une véritable redécouverte du "moi». Soumettre des extraits de cours aux enseignants, bouleverse en quelque sorte leurs habitudes et peut faire émerger des appréhensions liées à l'exercice. II y a d'abord l'image de soi qui semble "étrange " puisque la vidéo agit en miroir avec la particularité de pouvoir se voir faire, se voir dire et se voir se déplacer. Comme le signale Azaovi, "en favorisant l'explicitation de son action, le travail d'autoscopie conduit [l'enseignant] à prendre progressivement conscience de son identité professionnelle en action. II s'agit d'une nouvelle expérience à soi » (2014: 130). Cette expérience permet, selon (Cicurel, $2015: 43)$ :

d'aller au plus près de ce qu'on pourrait appeler la pensée-enseignanteen-action. Et, en effet, le flux de paroles que provoque le visionnement de l'action d'enseigner est révélateur d'une partie de ce qui habite l'univers intérieur du professeur. Les verbalisations ne sont pas la "réplique " des situations originales de classe de langue mais « la relation qui en est faite ».

\footnotetext{
9 II existe effectivement une sorte d'alimentation (par l'image) et de rétroalimentation (par l'image et par la parole).
} 
C'est la manière dont les acteurs se représentent l'action accomplie qui est porteuse de sens.

Dans les discours produits en autoconfrontation, nous avons pu dégager certaines particularités ou singularités chez les enseignantes. Nous avons remarqué que certaines verbalisations, souvent accompagnées de discours évaluatifs, montrent à quel point l'observation de soi permet de prendre conscience ou même de reprendre conscience de paramètres propres à des pratiques et façons de faire. En somme, les entretiens d'autoconfrontation, par l'intermédiaire du visionnage de soi, permettent l'émergence de logiques d'action, d'abord invisibles pour l'observateur qu'est le chercheur et souvent non conscientisés par les propres enseignants. Se dégage alors pour la compréhension de la pratique prescriptive, un regard personnalisé de cette pratique quotidienne du métier enseignant. Ces résultats contribuent à la compréhension de savoirs pratiques propres à l'agir professoral.

\section{Bibliographie}

ANADÓN, M. et F. GUILLEMETTE (2007): "La recherche qualitative est-elle nécessairement inductive? 1 . Recherches qualitatives. Hors Série, 5 : Actes du Colloque Recherche qualitative: Les question de l'heure. Disponible à l'adresse : http://www.recherche-qualitative.qc.ca/documents/files/revue/ hors_serie/hors_serie_v5/anadon.pdf.

AZAOUI, B. (2014) : "Quels apports de la vidéo en formation des enseignants de français? ». Diversité, SCEREN (CNDP-CRDP), pp.130-134, <halshs-01111818>.

BIGOT, V. et L. CADET (2011): "Comment la prise en compte des discours d'enseignants sur leurs pratiques renouvelle-t-elle l'analyse des interactions didactiques en classe de langue? ". Discours d'enseignants sur leur action en classe. Enjeux théoriques et enjeux de formation. Paris: Riveneuve éditions, pp. 11-29.

CICUREL, F. (2011) : Les interactions dans l'enseignement des langues. Agir professoral et pratiques de classe. Paris : Didier.

- (2015) : «Vers une conceptualisation de l'action en didactique des langues ». Le français aujourd'hui, 188, pp. 41-52.

CLOT, Y. (2001) : « Clinique du travail et action sur soi ». J.-M. BAUDOIN et J. FRIEDRICH (éds.) : Théories de l'action et éducation. Bruxelles: De Boeck, pp. 255-277.

- (2005) : « L'auto-confrontation croisée en analyse du travail : l'apport de la théorie bakhtinienne du dialogue \%. L. FILLIETTAZ et J. P. BRONCKART (dirs.) : L'analyse des actions et des discours en situation de travail. Louvain-la-Neuve : Peeters.

CLOT, Y. et D. FAÏTA (2000) : "Genres et styles en analyse du travail. Concepts et méthodes ». Travailler, 4, pp. 7-42.

COLETTA, J-M. (2005) : «Communication non verbale et parole multimodale: quelles implications didactiques? ॥. Le Français dans le monde. Recherches et Applications, Juillet, pp. 32-41.

COSNIER, J. et J. VAYSSE (1997) : «Sémiotique des gestes communicatifs ». Nouveaux actes sémiotiques, 52-54, pp. 7-28.

DOMINGUEZ, E. (2013): L'enseignant de FLE et l'activité de prescription: multimodalité (pré)réfléchie? Paroles sur l'agir professoral [Mémoire de master]. 
Angers: DUNE - Université d'Angers. Disponible à l'adresse : http://dune.univangers.fr/fichiers/20097748/20132MDLA1555/fichier/1555F.pdf.

DOMINGUEZ, E. et V. RIVIĖRE (2015) : "Les consignes en classe de langue : activité polyfocalisée et rôle du regard. Quels apports des discours réflexifs et quels enjeux de formation ॥. Les Cahiers de l'ACEDLE, vol. 12: La fabrique de l'action enseignante, $\mathrm{n}^{\circ} 2$.

RIVIÈRE, V. (2006): L'activité de prescription en contexte didactique. Analyse psycho-sociale, sémio-discursive et pragmatique des interactions en classe de langue étrangère et seconde [Thèse en élaboration; Université Paris 3 Sorbonne Nouvelle].

- (2008) : «Dire de faire, consignes, prescriptions... Usages en classe de langue étrangère et seconde ॥. J.-P. CUQ et F. DAVIN-CHNANE (éds.) : Le Français dans le monde. Recherches et Applications, 44, pp. 51-59.

VERMERSCH, P. (2004): "Aide à l'explicitation et retour réflexif ». Éducation Permanente, 160, pp. 71-80. 